Article

\title{
Enhancing the Skill of Geometric Prediction Using Dynamic Geometry
}

\author{
Elisa Miragliotta ${ }^{1, *(\mathbb{D})}$ and Anna E. Baccaglini-Frank ${ }^{2}$ (D) \\ 1 Department of Mathematics “F. Casorati”, University of Pavia, 27100 Pavia, Italy \\ 2 Department of Mathematics, University of Pisa, 56127 Pisa, Italy; anna.baccaglinifrank@unipi.it \\ * Correspondence: elisa.miragliotta@unipv.it
}

Citation: Miragliotta, E.;

Baccaglini-Frank, A.E. Enhancing the Skill of Geometric Prediction Using

Dynamic Geometry. Mathematics 2021,

9, 821. https://doi.org/10.3390/

math9080821

Academic Editor: Michael Voskoglou

Received: 1 February 2021

Accepted: 6 April 2021

Published: 9 April 2021

Publisher's Note: MDPI stays neutral with regard to jurisdictional claims in published maps and institutional affiliations.

Copyright: (c) 2021 by the authors. Licensee MDPI, Basel, Switzerland. This article is an open access article distributed under the terms and conditions of the Creative Commons Attribution (CC BY) license (https:/ / creativecommons.org/licenses/by/ $4.0 /)$.

\begin{abstract}
This study concerns geometric prediction, a process of anticipation that has been identified as key in mathematical reasoning, and its possible constructive relationship with explorations within a Dynamic Geometry Environment (DGE). We frame this case study within Fischbein's Theory of Figural Concepts and, to gain insight into a solver's conceptual control over a geometrical figure, we introduce a set of analytical tools that include: the identification of the solver's geometric predictions, theoretical and phenomenological evidence that s/he may seek for, and the dragging modalities s/he makes use of in the DGE. We present fine-grained analysis of data collected during a clinical interview as a high school student reasons about a geometrical task, first on paper-and-pencil, and then in a DGE. The results suggest that, indeed, the DGE exploration has the potential of strengthening the solver's conceptual control, promoting its evolution toward theoretical control.
\end{abstract}

Keywords: geometric prediction; conceptual control; Dynamic Geometry Environment (DGE); phenomenological evidence; theoretical evidence

\section{Introduction: Geometric Prediction and Dynamic Geometry as Resources for Supporting Mathematical Reasoning}

Prediction is the act of anticipating the outcome of a process, building on information at one's disposal at that moment. Research in science education highlights the fruitful practice of asking students to make predictions when they are learning about new phenomena (see, for example, [1]). Although the process of prediction has not yet been widely investigated within Mathematics Education, some studies have suggested that prediction can play an important role in this discipline, as well [2]. According to Kasmer and Kim [3], mathematics teachers create precious learning opportunities when they ask students for a prediction at the beginning of problem-solving activities. Indeed, prediction seems to stimulate processes of visualization, sense-making, and creating connections between previously learned mathematical concepts and new ones that can be developed as the prediction occurs [3]. Making a prediction yields the opportunity to acquire new knowledge; therefore, gaining further insight into processes of prediction in mathematics seems to be a promising direction in Mathematics Education, as well.

The pioneering studies presented above have been carried out mainly within the domains of algebraic and arithmetical reasoning. Since less interest has been devoted to prediction within the domain of geometrical reasoning, which we call geometric prediction, our study focuses on the domain of 2D Euclidean Geometry. Geometric prediction appeared in [4] and then was widely investigated in [5,6]; further details are provided in the next section.

Moreover, prediction is quite relevant from an educational point of view, since it turns out to be crucial for fostering students' mathematical reasoning [7]. In this sense, geometric prediction can be seen in coordination with other mathematical activities such as conjecturegeneration and argumentation. Following Kim and Kasmer [7]: "Although prediction is often based solely on initial thoughts using prior knowledge, prediction sets a foundation 
for later discourse that gives students the opportunity to defend or refute ideas that they brought forth at the beginning of the exploration" [7] (p. 298). So, prediction can also be seen as a particular and initial process of conjecture-generation. Since mathematical activity is strongly intertwined with processes of conjecture-generation, visualization, gaining new insight into a given problem, and argumentation, as researchers in Mathematics Education, we are interested in learning more about prediction because it is involved in each of these processes [3-7].

The studies presented above have substantiated the significance of using prediction within the classroom practice to facilitate students' learning [1-3,7]. In particular, White and Gunstone have introduced an instructional sequence they refer to as prediction-observationexplanation (POE) [1]. In POE much attention is devoted to the design of tasks for students: students are required to make a prediction, observing or performing an experiment, and finally to reconcile any discrepancies between prediction and observation. POE was revealed to be particularly effective for teaching physics at school [1]. Moreover, the authors claimed that POE can be applied also to other domains, including mathematics; in this case, the procedure needs to be adequately adapted [1]. However, this issue was not further discussed by the authors. Our paper addresses this issue by describing the specificity of producing and checking prediction in the field of geometrical reasoning. Indeed, the POE procedure seems to be quite relevant in geometry, as well, if a Dynamic Geometry Environment (DGE) is involved in the POE sequence. The exploration within the DGE can be considered quite close to the observation phase of a phenomenon (here the phenomenological behavior of the dynamic objects), which can be useful for testing the coherence of previously made predictions and, eventually, for changing or refining them. After all, the crucial role that the interaction between the students and a dynamic figure in a DGE plays in developing and supporting mathematical reasoning is widely recognized (see, for example, [8-15]). So, geometric prediction-as an initial stage of a conjecturegeneration process and a fundamental element of mathematical reasoning-could also be affected by the use of a DGE. Indeed, Hollebrands found that solvers' strategies used during a dynamic exploration "seemed connected to their ability to anticipate a result of a technological action and reflect on and interpret the result using their understanding of the technology tool and of mathematics" [16] (p. 184). So, the DGE's feedback plays a central role; such feedback might prompt solvers' next actions and lead them to eventually "change their strategy" [16] (p. 188). This "ability to anticipate" in geometry seems quite close to the process of geometric prediction, on which we focus; however, there is still very little research on the possible relationship between geometric prediction and DGE explorations.

Our study aims to start bridging this research gap by exploring how students' interactions with a DGE may influence their predictions made during an initial problem-solving phase prior to entering the DGE. In particular, the analyses of the case study discussed in this paper show that the interaction with DGE objects can lead to rethinking and renegotiating predictions that were previously communicated. Moreover, for this study we designed a theoretical lens for observing and explaining why this change can occur. This paper should provide educators with food for thought and support them in establishing educational goals in learning geometry that capitalize upon the combined use of predictions and DGE explorations. The analytical tools developed for this study are presented in depth, so we expect that other researchers may apply them in the future. Overall, the study contributes to the line of research opened by $[5,6]$ and allows us to advance new hypotheses to be further explored.

This paper is structured as follows. In Section 2, we outline the theoretical framework, and we present our research aim; in Section 3, we describe materials and methods, specifically focusing on the analytical tools developed for this study; in Section 4, six excerpts from a case study are presented and analyzed in fine-grained detail, in order to address our research aim; finally, Section 5 provides a discussion of the main findings and reports on educational implications and future perspective of our research. 


\section{Literature Review, Theoretical Grounding of the Study and Specific Research Aim}

Our interest in geometric prediction arose during a transdisciplinary (in the sense of [17]) attempt to integrate research on the transformation of images that have been developed within two different research domains: Cognitive Psychology and Mathematics Education. More precisely, our purpose was to shed light onto processes of transformations of geometrical objects (e.g., rotation, translations, reflection, stretching, etc.) during geometrical activities [18]. This first research study gave us the opportunity to observe a common phenomenon: solvers, who engage in the resolution of open-ended tasks involving a geometrical configuration, can anticipate the effects of certain transformations before they were accomplished on a sheet of paper or within a DGE. We have referred to this phenomenon as geometric prediction (GP). Our findings $[5,6,18]$ suggest that mental transformations of geometrical configurations during a geometric prediction not only involve elements of spatial nature, but also aspects that belong to the mathematical reference theory. So, attempting to conduct a second transdisciplinary inquiry on prediction, we worked to find a definition of geometric prediction that took into account both spatial and theoretical aspects involved in doing geometry. The definition (see Section 2.1) and the model of geometric prediction that arose are heavily rooted in this background and have been presented by the first author [6].

In the following we will clarify the distinction between spatial and theoretical aspects, and we will present the fundamental theoretical constructs used in this study.

\subsection{Geometric Prediction through the Lens of the Theory of Figural Concepts}

Cognitive psychologists explain the processes of generation, transformation, and use of mental images with the intervention of visuo-spatial abilities [19]. More recently, these abilities have been explicitly related to geometrical reasoning [20]. Our greater concern is that this psychological approach alone only addresses the spatial and visual aspects of geometrical objects [21]. As a consequence, it does not properly consider the conceptual essence of those objects as part of a formal mathematical theory. This point of view is in line with recent considerations on spatial ability [22].

On the other hand, literature in Mathematics Education has frequently addressed the issue of the tension between the spatial aspects and the logical structure of geometry (for an extensive review of the literature from 2008, see [23]; before that, see, e.g., [24-26]). As other mathematical domains, geometry is a logical system made up of definitions and theorems, and whose objects are ideal. Geometry, as a mathematical theory, is a cultural artifact that, at the beginning, relies on a more natural conceptualization of space; however, also at a more advanced stage - when geometry acquires a logical conceptualization-its objects can continue sharing attributes of both conceptions of geometry [24]. This is the key reason why, when we want to investigate students' personal use of and interaction with geometrical objects from an educational point of view, we need a theory that describes the multifaceted nature of geometrical objects and that offers a lens to look at how their different components are intertwined. Fischbein's Theory of Figural Concepts [27] does exactly this.

Indeed, Fischbein considered geometrical objects as having a dual nature: as concepts they are completely described and controlled by an axiomatic system of definition and theorems, but at the same time they maintain certain figural aspects of images. When a solver is approaching a geometrical task, the distinction between images and concepts is not so clear cut, and the solver deals with "a third type of mental objects which simultaneously possess both conceptual and figural properties" [27] (p. 144). This hybrid entity is called a figural concept; it is neither a pure concept nor a pure image, it is the fusion between the conceptual and the figural components of a geometrical object as it has been thought by the solver. In Fischbein's words: figural concepts "reflect spatial properties (shape, position, magnitude), and at the same time, possess conceptual qualities-like ideality, abstractness, generality, perfection" [27] (p. 143). Seemingly, a geometrical figure is a mental image controlled by a definition and directly connected with a figural concept; the drawing is 
only a material embodiment of the figure. In this paper, we will use the term "figure" or "geometrical figure" in this sense.

Consequently, we can recognize and distinguish between the figural components and the conceptual components of a geometrical figure or a figural concept.

- The conceptual components refer to the theoretical status of a figural concept: the definition, the properties, the theorems, as they were learned and conceptualized by the student. They may be affected by logical fallacies [27] (p. 145). In this paper, the conceptual components are part of the solver's Theory of Euclidean Geometry (TEG) or, more precisely, of the solvers' conceptualization of the TEG.

- The figural components refer to the spatial properties of a figural concept. They may be influenced by the Gestalt theory of perception or graphical constraints [27] (p. 160).

For example, when a student is constructing the figural concept of rectangle, she could conceptualize some properties as a concept, like the relationship of parallelism or perpendicularity, and some others as figural properties that belong to concrete experience, like the "rectangularity," referring to the rectangular shape of a table or a notebook or a window.

So, according to Fischbein's interpretation, from a psychological and educational point of view, when people use or refer to geometrical objects they are thinking in terms of figural concepts. Since figural concepts are individually constructed and evolve during the learning experiences, students' figural concepts can be very close or quite far from the corresponding geometrical objects.

Using the theoretical framework described above, geometric prediction can be defined as follows. Geometric prediction $(G P)$ is the cognitive process of generation of a new geometrical object through the manipulation of its figural elements that maintain invariant certain theoretical elements belonging to the solver's Theory of Euclidean Geometry (TEG). We refer to the outcome of a GP process as a product of GP.

As remarked by Mariotti and Baccaglini-Frank [4], GP can be seen as a skill of producing prediction that is supported by visuo-spatial abilities coupled with the monitoring of the theoretical consistency with the solver's TEG.

So, following the definition, the manipulations that lead the solver to a product of GP are accomplished within the figural domain, but under the monitoring of the solver's conceptual and theoretical control, which will be discussed in the next section.

\subsection{Conceptual and Theoretical Control}

Since the figural concept is an "image intrinsically controlled by a concept" [27] (p. 160), in order to consider the image of a geometrical configuration as a figural concept, the student needs to see such an image in coordination with its geometrical definition. Moreover, as s/he solves the task, the solver can interact with the figural components of the figural concept by manipulating and transforming them. Fischbein, followed by Mariotti, described a system of control of the conceptual consistency of the manipulation that a solver accomplishes on a geometrical configuration: the conceptual control. For example, when the solver needs to rearrange figural components of a given drawing (for example, through rotation, translation, reflection, etc.), "the conceptual control system can affirm the possibility and the correctness of this procedure" [28] (p. 15).

In addition, conceptual control is responsible for impeding the figural components from behaving autonomously. Indeed, very often the figural components dominate the interpretation of a drawing of a geometrical figure. This can lead to an interpretation that is consistent within the figural domain, but completely incoherent with the conceptual constraints within the reference mathematical theory. This case can be described as having figural components that escape the conceptual control.

Nevertheless, since the students' conceptualization of geometrical objects can be more or less consistent with the TEG, the solver's conceptual control may be more or less close to the theoretical control, the act of "mentally imposing on a figure theoretical elements that are coherent in the theory of Euclidean geometry" [4] (p. 156). When a solver's 
conceptualization is fully Euclidean, s/he can use theoretical control to dominate the figural components. The two constructs of theoretical and conceptual control are different essentially because: theoretical control is always coherent with the reference theory; on the other hand, conceptual control is coherent with the solver's personal mathematical theory, which may not be consistent with the theory of reference.

\subsection{DGE and Paper-and-Pencil Environment}

Since GP processes are strongly influenced by the solver's conceptual control [4-6], we are interested in gaining a deep insight into possible transitions from conceptual to theoretical control. Such transitions-if they occur-will be key in determining the solver's potential learning.

DGEs like Cabrì Géomètre, Geometer's Sketchpad, GeoGebra or Desmos consist of digital artifacts that represent geometrical objects (like points, lines, circles, and so on) and that can be constructed and transformed according to (for example, but not only) the axioms and theorems of Euclidean Geometry. In Lopez-Real and Leung's [29] words: "It is a virtual mathematical reality where abstract concepts and constructions can be visually reified" (p. 665). In this sense, geometrical objects become phenomenological entities that a solver can observe and figurally transform.

The dynamism of DGE objects constitutes one of the main features of DGEs. Dragging is the function that allows direct manipulation of the objects on the screen [11], inducing transformations that can be perceived as movements of the same object. Moreover, solvers can perceive a dynamic object as a dynamic figure (i.e., a geometrical figure within these microworlds): the DGE object phenomenologically embodies the figural components of the figure, and the solver might project onto the dynamic figure conceptual components of the corresponding geometrical figure. This interpretation depends on the solver's conceptual control exercised upon the figure. However, a DGE object has specific features: it satisfies the set of theoretical conditions used for constructing the object within the DGE; it satisfies and maintains all the theoretical constraints that logically derive from the given ones. Indeed, the theoretical constraints are maintained invariant also when the solver figurally changes the dynamic figure using the drag modality. From an educational point of view, this is an interesting aspect. Indeed, a figure on a DGE screen shows the figural components of a geometrical object, but at the same time it maintains a strong and coherent connection with its theoretical components. The figural components cannot escape a theoretical control that is ever consistent with the formal TEG. In this sense, the figural components of a dynamic figure are always under the theoretical control exercised by the software.

Now it is clear that when a solver approaches a geometrical problem within a DGE, the context is different from the paper-and-pencil environment. In a paper-and-pencil environment when a solver approaches a geometrical problem s/he sketches it out, and manipulates the figure, either mentally or tracing out another drawing of the same situation. During this process, in order to maintain invariant the theoretical elements of the geometrical configuration, the solver must keep track of them and make sure that these are all present in the new drawing [14] by exercising conceptual control (as coherent as possible to the TEG) over the figure. This can be quite challenging for students. On the other hand, in a DGE, transforming a figure can be performed simply by dragging vertices of the figure; automatically, the DGE object maintains all the properties according to which the original figure was constructed (and all of their consequences deducible through theorems in the TEG). Therefore, the solver does not have to keep track of the figure's conceptual components, but s/he can concentrate on a specific figural component or theoretical constraint, resting assured that all the other constraints will remain invariant. Shortly, as stated by Laborde [30] (p. 172), "the computer not only enlarges the scope of both possible experimentation and visualization but modifies the nature of the feedback. The feedback is visual on the surface, but it is controlled by the theory underlying the environment." In other words, the feedback is always coherent with the TEG both in its theoretical and figural components. 
In conclusion, clearly the nature of interactions with a static drawing or with a DGE object is different: while on paper, checking the coherence of figural transformations with the TEG is up to the solver, in a DGE such a responsibility can be offloaded onto the software. We are interested in exploring how this fundamental difference between these two contexts can influence (and possibly foster development of) students' conceptual control involved in GP processes. Before further elaborating on this research aim, we introduce the notions of phenomenological and theoretical evidence.

\subsection{Evidence}

When interacting either with a figure on paper or with a dynamic figure to generate conjectures (GPs can be seen as a particular kind of conjecture, as we mentioned in the introduction) the solver is frequently trying to identify or produce information to support his/her reasoning. Such reasoning can be broken into implicit claims related to the conjecture being generated. This process of producing, confirming or rejecting implicit claims is closely connected to the solver's generation of evidence for such claims. Building on Baccaglini-Frank's work, by evidence we intend "the available body of facts or information indicating whether a belief or proposition is true or valid" [31] (p. 779).

In the domain of Euclidean Geometry, the validity of a proposition is given by a body of facts, such that subsets of them can be organized into deductive chains that prove the proposition within the underlying theory. However, the definition also "implicitly relates such a 'body of facts' to a person, an actor, to whom the facts are available and for whom they indicate validity or truth of the proposition" [31] (p. 780). Moreover, Baccaglini-Frank, elaborating on the notion of evidence in the context of conjecture-generation supported by a DGE, distinguished two forms of evidence [31] (p. 786):

- Theoretical evidence, that is, facts referred to a mathematical theory; they can be sufficient to be organized into deductive chains proving a proposition in Euclidean Geometry; or they can be insufficient for reaching a proof;

- Phenomenological evidence, that is, information collected as feedback from the DGE (or the drawing on paper) indicating the truth of a proposition.

Indeed, within the phenomenological domain of a DGE, claims may be perceived as true based on the facts that the solver collects as feedback from the system [31] (p. 780), where figures on the screen have a physical nature, as they can be interacted with through physical movement.

Similarly, within the paper-and-pencil environment a solver can gather evidence from his/her drawings by producing several examples or counterexamples for the claim. However, as discussed above, the generation of a new coherent drawings is strongly determined by solver's conceptual control.

We are interested in analyzing what forms of evidence a solver uses to support or reject a claim in order to gain further insight into his/her conceptual or theoretical control during the problem-solving process.

\subsection{Research Aim}

The general aim of this paper is to qualitatively analyze some possible roles of a DGE in a student's prediction processes previously carried out in a paper-and-pencil environment. Since this is a very general and ample purpose, we concentrate on a single aspect. More precisely, our focus is on conceptual and theoretical control: we will study how a DGE exploration that happens after processes of GP can influence the solver's conceptual control.

In the next section, we will describe the materials and methods of the study, highlighting our analytic procedure for making inferences on solvers' conceptual control from the data collected. 


\section{Materials and Methods}

This section comprises several parts: in the first we describe the design of the study and provide information about the solver, who is the protagonist of the case study; in the second, we present the task upon which this study is focused and a general description of prediction open problems; in the third, a wide description of the analytical tools designed for this study is given.

\subsection{Design of the Study}

In this paper we present a case study involving a 9th-grade high school student. The overarching project in which the present case study is embedded aimed at investigating some cognitive aspects involved in the GP processes. Specific tasks were designed in order to elicit and observe GP processes, during semi-structured task-based interviews [32-34]. Tasks had a common structure that was composed of two parts as described below.

- The interviewer explained the geometrical situation, showing the task written out on paper. The task had to be performed imagining or drawing the geometrical configuration in a paper-and-pencil environment.

- Once the solver had proposed a solution or stated that she was not able to find one, the interviewer opened a DGE file and asked the solver to move some points of the dynamic figure, in the same way as in her prediction, and to explore it with the intent of reaching another or more complete solution.

Further details on the tasks designed are presented in the next section.

Data were collected during individual task-based interviews [33] conducted by the first author during the months of February and May 2018. All interviews were carried out in a quiet room and each solver spent $60 \mathrm{~min}$ with the interviewer, working through as many tasks as s/he could. In this paper we focus on a single task (see Section 3.2). The interviewer was not a teacher for these solvers; she interacted with them once before the interviews, only to introduce herself and gather the volunteers. The interviews were video-recorded using a camera next to the solver. Data included: video recordings, audio recordings, transcriptions, solvers' drawings. The participants' parents or guardians signed a consent form concerning the use of data for scientific purposes. Moreover, all the schools the participants attended had specific informed consent forms approved by their administration, which had been signed at the beginning of the year by the parents of the students.

The explorative nature of our research aim led us to use a case study approach. This qualitative methodology is particularly fruitful for analyzing how a certain phenomenon is influenced by the particular context where it occurs [35]. So, it was particularly indicated to unveil how a dynamic exploration within a DGE that follows a paper-and-pencil approach to the task (the context) influenced GP processes (the phenomenon). Since our intent was to gain insight into this particular situation, we implemented an instrumental case study [35]: the solver's interview during the resolution of the task was the unit of analysis; the case played a supportive role in describing the possible influence of a DGE exploration. This methodological choice allowed us to look at the case in depth [35]. We conducted our qualitative analysis according to the analytical procedure that we will describe in Section 3.3. Some rounds of analysis were independently conducted by the researchers (the authors of this paper) and then discussed together.

For this case study we chose Ilaria (a pseudonym). When the interview took place Ilaria was a 9th-grade student; her mathematics teacher had introduced GeoGebra and used it only for producing fixed step-by-step constructions. In Ilaria's class students had never been invited to drag points or move the figure to produce conjectures. As regards geometry, at the beginning of high school, the teacher introduced, among other concepts, the classification of triangles, the midpoint and the perpendicular bisector of a segment. In particular, based on the mathematics curriculum of her previous classes, which followed the Italian National Guidelines for Instruction [36], Ilaria had been exposed to the Pythagorean theorem, and she knew that a right triangle can be inscribed into a circle. Moreover, she 
had been presented with the definition of a circle as the locus of points that are equidistant with respect to a fixed point, and with the relationships of perpendicularity and parallelism between lines on the plane. To be sure of the curriculum covered, we also interviewed her mathematics teachers about the mathematical facts that Ilaria was supposed to know.

We chose Ilaria as our case to study because, on the one hand, during the interview she was quite talkative and produced several gestures and drawings on her own with minimal intervention on behalf of the interviewer, so the excerpts were quite rich in terms of productions to be analyzed; on the other hand, Ilaria's interview was carried out in a relatively short time compared to most of the other participants, making it ideal for being reported in a paper.

\subsection{Materials}

This paper focuses on the following task, since it had not yet been discussed in other publications about the greater study within which our data were collected.

Consider the right triangle in the figure (Figure 1), with the hypotenuse of fixed length. $\mathrm{A}$ and $\mathrm{B}$ are fixed. The length of $\mathrm{AB}$ must always be the same.

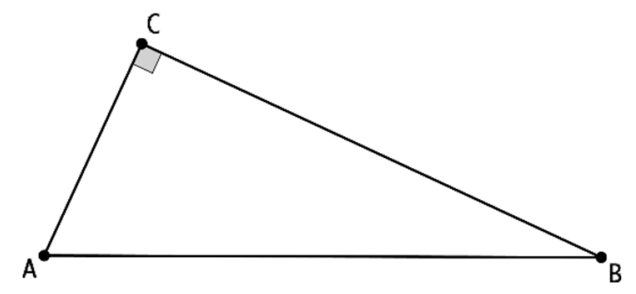

Figure 1. The drawing provided by the task.

What can you say about the vertex with the right angle?

We were interested in how a solver could freely explore the situation to reach possible solutions. The task provided an example of prediction open problem. The expression open problem $[37,38]$ refers to a task stated in a form such that the solvers do not have specific instructions to be followed: they are left free to explore the problem and draw their own conclusions. The question does not suggest, reveal or anticipate the solution or a possible answer. Particularly in geometry, the literature has discussed the general structure of an open problem [39]: it is not a problem reduced to a mere implementation of an already known procedure or routine. The solver is asked to carefully choose a solution path and make a conjecture, which may not be unique. Often the resolution process can lead the solver to the formulation of a conjecture expressed as a conditional statement after a (physical or mental) exploration of the situation [40].

Prediction open problems are a particular kind of open problem in which the solver is asked to describe possible alternative arrangements of a geometric configuration (imagined, given by a drawing and/or by a step-by-step construction) maintaining given properties. More specifically, we decided to construct prediction open problems that provided a stepby-step construction to be imagined or accomplished on a sheet of paper. This design choice was made because we wanted the solvers to be able to freely access the theoretical elements that characterized the geometrical configuration to be reasoned upon.

Moreover, we designed the step-by-step constructions so that the dynamic figure, which the solvers would use during the exploration of the problem within the DGE, corresponded to the given or sketched out figure. For this reason, the step-by-step constructions were designed in the DGE to maintain the constraints of the problem robustly [41] (the triangle with the fixed hypothenuse in the selected problem), while the properties that the solver needed to recognize and maintain for coherently solving the problem were constructed softly (the right angle in the selected problem).

According to the principle of a semi-structured interview [32-34], the first question is always the same, but the interviewer has a sequence of specific questions defined a priori 
and a set of general stimuli to obtain solvers' comments or clarifications. The possible additional specific questions for this task were the following:

1. Make a prediction: do you think that it (or point $C$ or the vertex with the right angle) can occupy other positions?

2. Make a prediction: do you think that it (or point $C$ or the vertex with the right angle) can occupy other positions so that the angle stays right?

3. Imagine moving the vertex $C$ and make a prediction: do you think that it can occupy other positions so that the angle stays right?

For each of these questions: if the solver's answer was "Yes", the interviewer asked "Which?" or "How?"; if the solver's answer was "No", the interviewer asked "Why not?"

In general, we adopted a non-interventionist approach: when the solvers communicated contradictory answers or products of GP that were incoherent with respect to the formal TEG, we did not point out to the solvers the contradictions of their answers.

\section{Data Presented}

Excerpts of the case study were selected in order to give in a quite short time span a telling example of the evolution of the solver's focus, generation of evidence, and processes of prediction. We chose Ilaria because in less than $10 \mathrm{~min}$ she communicated several products of GP and reached a satisfactory (for her) solution to the problem. Moreover, excerpts show that Ilaria's predictions actually changed during the DGE exploration, as did her conceptual control. So, these excerpts provided useful data for analyzing in depth the specific contribution of the DGE exploration. The excerpts selected provided an accurate overview of Ilaria's prediction generation.

\subsection{Analytic Procedure}

According to the explorative nature of our research aim, we chose a qualitative approach for data analyses.

Each interview was transcribed from the videos. The interviews were conducted in Italian and then translated into English for the present study. We also took into account the solver's behavior and her non-verbal productions, including gestures, drawings, and long silences.

After the transcription phase, in the first round of analysis we identified instances of GP in the paper-and-pencil environment. To recognize the product of a prediction process we identified instances at which: the solver referred to elements (a geometrical object or part of it) that were not present in the drawing; or the solver described the behavior of elements that were not present in the drawing; or the solver referred to a new arrangement of the configuration without drawing anything. This first phase led to the identification of the solver's GP processes, which we expected the subsequent DGE phase to refer to.

In the second round of analysis we focused on the solver's work in the DGE, singling out, with reference to the GP processes identified in the first round of analysis, new GPs or modifications of those previously made.

Moreover, analyzing the solver's utterances and gestures performed during both phases, we identified the main claims (be they explicit or implicit) for which the solver seemed to be generating evidence. The claim was reported using as much as possible the solver's verbal productions; on the other hand, the inferred products of GP were formulated in a short sentence by us.

The reference to a specific claim determined the composition of the excerpts to be analyzed during the third round. For each excerpt, which constituted a unit of analysis through the case study, we considered the following aspects:

- Words said by the interviewee,

- Gestures and drawings made by the interviewee,

- (Possible) sub-products of GP, leading to the main GP and identified through a "spotlight,"

- The claim for which the solver searches,

- Evidence that will be characterized as phenomenological or theoretical. 
Further details on the operative identification of instances of GP and evidence are provided in [6] and [31], respectively. We made use of these observable aspects for collecting interpretative elements that supported inferences and considerations about the solver's conceptual control. Indeed, from a cognitive point of view, conceptual control is a complex construct that is not directly observable (at least so far).

While we already introduced what we mean by GP and how we identified the products of such a process in the interview excerpts, we now explain what we mean by spotlight, and how dragging modalities allowed us to make inferences about the solver's generation of evidence.

The Oxford Dictionary defines the noun spotlight as "a light with a single, very bright beam that can be directed at a particular place or person"; metaphorically, we use the term to suggest that we are showing what (we hypothesize) the solver is focusing on. Exploiting this metaphor, in the analyses we will use an image that places at the illuminated center the part of the image (drawing or construction on the screen) that seems to be in focus for the solver, while the rest of the image is partly in a gray zone and then fades into the darkness. So the spotlight visually shows our interpretation of the figural components in focus for the solver. The spotlight can shine on greater or smaller areas of the figure, and the focus can be very narrow or quite wide. Coupled with the solver's gestures and utterances, the spotlight also allows us to interpret which theoretical properties the solver is directing his/her attention to. In return, the spotlight provides us further information on the evidence that the solver is gathering from his/her exploration.

Moreover, for each claim, the solver knows something (for example, conceptual and figural elements previously identified that have shaped the eventually implicit claim) and wants to learn more about such a claim by finding evidence, be it theoretical or phenomenological. Our working hypothesis was that, within the DGE, dragging modalities can provide a deep insight into the solver's processes of evidence generation and identification. Indeed, in a DGE, exploring a dynamic figure can become a search for invariant properties under dragging [30,42-45]. Previous research has analyzed the use of dragging from a cognitive point of view; see, for example, $[43,46,47]$ for identifying particular ways in which dragging seems to affect students' reasoning processes. In this study, we used the following dragging modalities first introduced by Arzarello and colleagues [43] and readapted by Baccaglini-Frank and Mariotti [47].

- Wandering dragging (WD): moving a point of a dynamic figure randomly, without a plan, or looking for interesting configurations;

- Maintaining dragging $(M D)$ : moving a point so that the dynamic figure maintains a certain desired property;

- Dragging test (DT): moving a point in a given way in order to see whether the figure maintains invariant a certain property.

From the way in which these dragging modalities are presented, it is clear how they open windows onto the solver's reasoning process: for example, to recognize $\mathrm{MD}$, we made an inference on the solver's intention as s/he acted on the DGE figure. Such an inference, in the current study, helped to interpret what part of the feedback obtained the solver recognized as evidence for such a GP from the DGE. Indeed, as perceptual feedback was generated by the DGE during dragging, the solver's interpretation of such feedback could influence her original product of GP.

\section{Results of the Analyses}

In this section we analyze the most significant aspects of Ilaria's exploration within the paper-and-pencil and dynamic geometry environments as concerned the shifts in her focus, the unfolding of her prediction processes, and her evidence generation. These aspects were identified based on the analytic procedure described in Section 3.3, and we used them to advance inferences about the solver's conceptual control over the figure. 


\subsection{Ilaria's Interview during the Resolution of the Given Task}

In the following sections, we present six excerpts from Ilaria's interview and the corresponding scheme of analyses. In particular, we analyzed two excerpts from the paper-and-pencil resolution and four excerpts from the subsequent DGE exploration.

\subsubsection{Ilaria's Paper-and-Pencil Exploration}

The first excerpt started right after the interviewer asked Ilaria to make a prediction about the right angle: Do you think that it can occupy other positions so that the right angle stays right? Ilaria stated that she thought so and explained why (Table 1); the main elements captured in the analytic process are presented in Table 2.

Table 1. Excerpt 1 from Ilaria's interview.

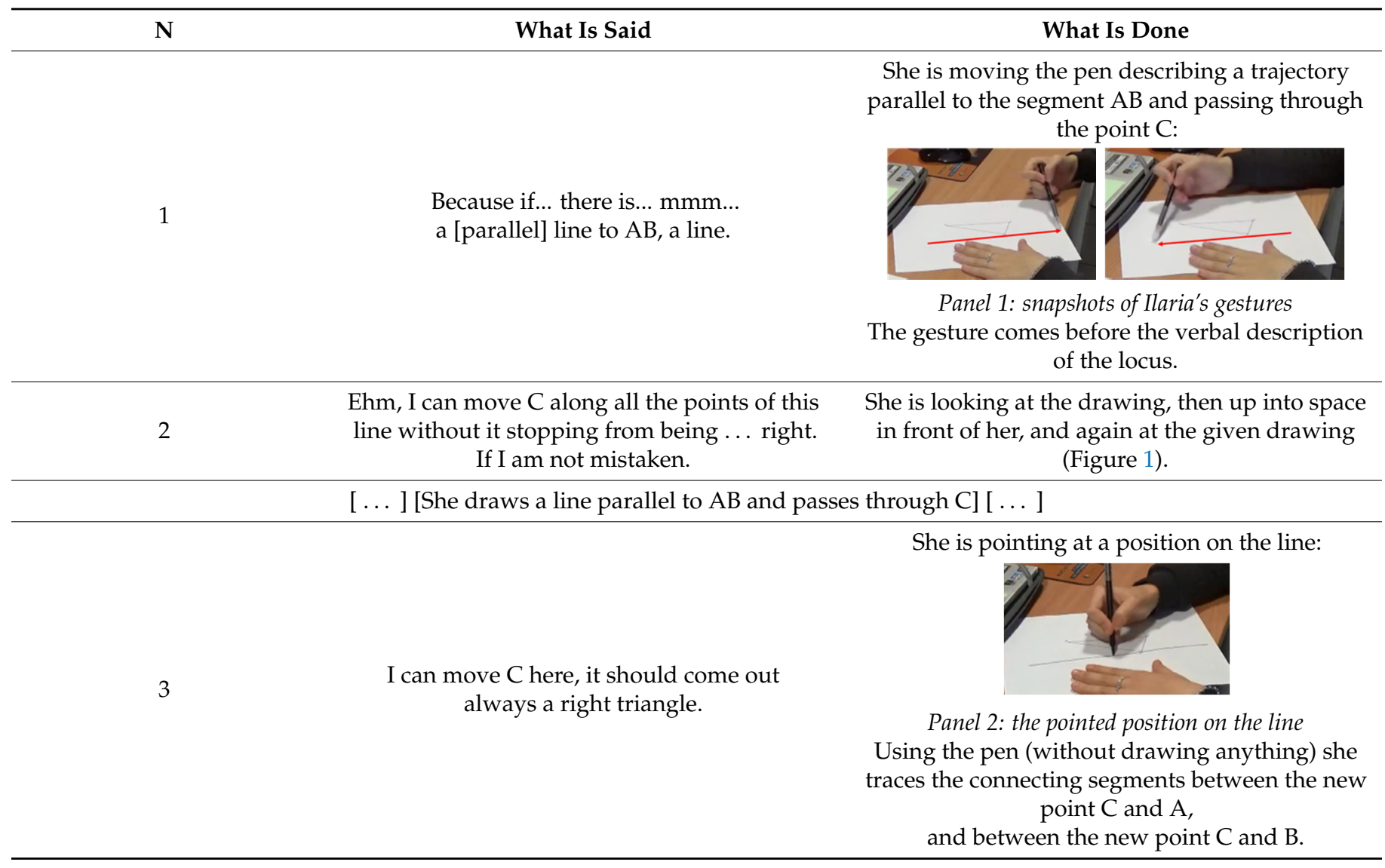

At the beginning, Ilaria introduced the new figural concept parallel line that was conceptualized and represented as a straight line passing through C (see Panel 1). Then the relationship between $C$ and the line changed: $C$ was reconceptualized as a point on the straight line ("C along this line"). Ilaria seemed to imagine a continuous movement of $C$ along the line, in positions near the one shown in the spotlight, not relating the dynamic vertex of the right angle to the rest of the triangle. The evidence gathered, if any, from this dynamic image seemed to be mostly phenomenological, that is, not supported by many theoretical elements. Nevertheless, this seemed a first attempt to generate theoretical evidence as she verbally described the movement of $C$ along a line: for example, her idea might have come from previous experiences with triangles with a varying perimeter but the same area, obtained by fixing a side and moving the free vertex along a line parallel to the fixed side. However none of Ilaria's utterances allowed us to confirm this hypothesis. 
Table 2. Main elements from the analysis of Excerpt 1.

\begin{tabular}{|c|c|c|}
\hline Labels & Name & Description \\
\hline \multirow{6}{*}{ Spotlight } & \multirow{3}{*}{ S_1 } & $\begin{array}{c}\text { The spotlight encompasses the parallel line and the right } \\
\text { angle. The content of the spotlight is: a straight line through } \\
\text { C, with the property of being parallel to AB; } \\
\text { the right angle at C. }\end{array}$ \\
\hline & & \\
\hline & & Spotlight 1: a picture of the inferred spotlight \\
\hline & \multirow{3}{*}{ S_2 } & $\begin{array}{l}\text { The spotlight is on the angle, while } C \text { is at specific positions } \\
\text { that are checked for providing "good examples" of the } \\
\text { claim. The spotlight seems to illuminate specific discrete } \\
\text { positions of C along the line, where the angle's sides seem to } \\
\text { be imagined (dotted red within the picture below). }\end{array}$ \\
\hline & & \\
\hline & & Spotlight 2a: an instance of the moving spotlight \\
\hline \multirow{4}{*}{ GP } & gp_1 & a straight line parallel to the segment $A B$ \\
\hline & gp_2 & $\mathrm{C}$ on the parallel line \\
\hline & GP_1 & $\begin{array}{c}\text { when } C \text { is on a straight line parallel to the segment } A B \text {, } \\
\text { the angle is right }\end{array}$ \\
\hline & gp_3 & the triangle is right when $C$ is close to the given position \\
\hline Claim & C_1 & $\begin{array}{l}\text { I can move } C \text { along all the points of this line [as in spotlight] } \\
\text { without [angle } C \text { ] stopping from being right. }\end{array}$ \\
\hline \multirow{2}{*}{ Evidence } & E_1 & $\begin{array}{l}\text { Mostly phenomenological: Ilaria attempts to generate } \\
\text { phenomenological evidence to confirm predictions using } \\
\text { her drawing. }\end{array}$ \\
\hline & E_2 & $\begin{array}{l}\text { Phenomenological evidence seems to be provided to Ilaria } \\
\text { as she points to positions of } C \text { with the tip of her marker. } \\
\text { According to Ilaria this evidence at first confirms the claim. }\end{array}$ \\
\hline
\end{tabular}

In this excerpt, Ilaria intended to find positions such that the angle was "always" right (utterance 3). Driven by gp_3, Ilaria seemed to have found an example that confirmed both the claim and her GP_1. Looking at our reconstruction of Ilaria's spotlight (Spotlight 2a), we noticed that this example did not correctly support her claim (one can easily recognize that the position of $C$ pointed to did not figurally produce a right angle); however, for Ilaria it seemed to provide phenomenological evidence that was coherent with the claim. From this episode we inferred that the solver's conceptual control over the figure was not fully Euclidean.

In excerpt 2, she started exploring her predictions (Table 3); the main elements captured in the analytic process are presented in Table 4. 
Table 3. Excerpt 2 from Ilaria's interview.

$\mathbf{N}$
What Is Said

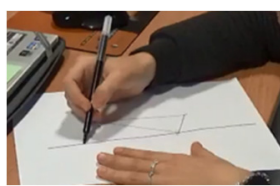

Panel 3: a new position on the line

She draws two segments:

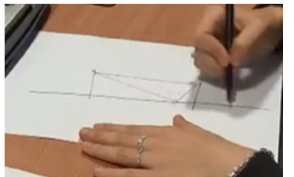

Panel 4: the moment when Ilaria has drawn two segments

\section{$[\ldots]$}

Moving it along this $r$, on this line $r$. . r...ehm...C... I can move on any point of this line $\mathrm{r}$... between the projections of...of $B$ and of $A$ on this line... and it should remain...C...and the angle $\mathrm{C}$ always right.
She focuses on a new drawing:

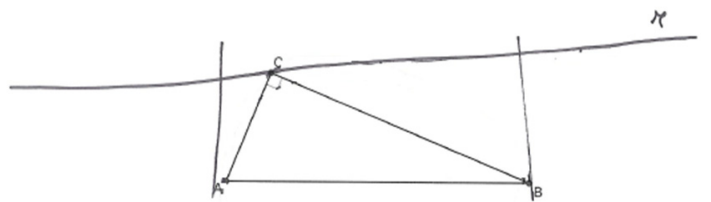

Panel 5: the new drawing upon which Ilaria is reasoning

Table 4. Main elements from the analysis of Excerpt 2.

\begin{tabular}{|c|c|c|}
\hline Labels & Name & Description \\
\hline \multirow{6}{*}{ Spotlight } & \multirow{3}{*}{ S_2 } & The same as above \\
\hline & & \multirow[b]{2}{*}{ Spotlight $2 b$ : an instance of the moving spotlight } \\
\hline & & \\
\hline & \multirow{3}{*}{ S_3 } & $\begin{array}{l}\text { The spotlight is on the vertices with the right angles on the parallel } \\
\text { segment contained between the projections. }\end{array}$ \\
\hline & & 1 \\
\hline & & Spotlight 3: the region of interest within the spotlight \\
\hline \multirow{3}{*}{ GP } & gp_4 & $\begin{array}{l}\text { the triangle is no longer right when } C \\
\text { is far away from the given position }\end{array}$ \\
\hline & gp_5 & two projections of $\mathrm{A}$ and $\mathrm{B}$ on the parallel line \\
\hline & GP_2 & $\begin{array}{l}C \text { on a line parallel to } \mathrm{AB} \text { and between } \\
\text { two projections of } \mathrm{A} \text { and } \mathrm{B} \text { on this line. }\end{array}$ \\
\hline \multirow[b]{2}{*}{ Claim } & C_1 & [same as above] \\
\hline & C_2 & $\begin{array}{c}\text { Moving }[\mathrm{C}] \text { along this line } \mathrm{r} \text {, between the projections of } \mathrm{B} \text { and of } \mathrm{A} \text {, } \\
\text { the angle } \mathrm{C} \text { [should] always [remain] right. }\end{array}$ \\
\hline
\end{tabular}


Table 4. Cont.

\begin{tabular}{|c|c|c|}
\hline Labels & Name & Description \\
\hline \multirow{3}{*}{ Evidence } & E_2 & $\begin{array}{l}\text { Phenomenological evidence seems to be provided to Ilaria as she } \\
\text { points to positions of } C \text { with the tip of her marker (Panel } 3 \text { and } \\
\text { Spotlight } 2 b \text { ). According to Ilaria this evidence contradicts in part the } \\
\text { original claim (C_1), leading to its revision (see utterance 6). }\end{array}$ \\
\hline & E_3 & $\begin{array}{l}\text { Phenomenological evidence: adding two orthogonal projections, Ilaria } \\
\text { searches for phenomenological evidence to support the new prediction. }\end{array}$ \\
\hline & E_4 & $\begin{array}{l}\text { Theoretical evidence: once the two segments are drawn, she adds a } \\
\text { reasonable theoretical counterpart, by describing them as projections. } \\
\text { In this way, she attempts to generate theoretical evidence, by trying to } \\
\text { recall an explanatory fragment of theory. }\end{array}$ \\
\hline
\end{tabular}

Looking for other evidence, the spotlight moved along the parallel line to positions of $C$ far away from the given one. This led to evidence contradicting the claim, and that brought Ilaria to restating it in a weaker form (utterance 4-5-6). From the excerpt we inferred that Ilaria intended to observe how the angle did not change while she was moving $\mathrm{C}$ along the line; however, she did not seem able to conceptually control at the same time the movement of $\mathrm{C}$ and the consequences of such movements on the triangle. This could be the reason why she decided to explore the configuration discretely, looking for "good examples" (utterance 3-4).

Ilaria's search for phenomenological evidence produced a seemingly unexpected outcome, leading to a revision of the claim (C_2). Once the projections were drawn, Spotlight 3 was focused on the segment between the two projections, ignoring $\mathrm{AB}$ and the rest of the triangle. This was consistent with GP_2 not being consistent with the TEG, even though Ilaria seemed to perceive phenomenological evidence for her new claim. Once again, Ilaria seemed to imagine the movement of $C$ along the line and in particular at three positions (determined by the given point in the middle and the two projected points), but she did not show theoretical control over the whole geometrical figure.

Ilaria's reasoning seemed to be rooted in her first conceptualization of and predictions about the configuration. Even if the counterexample disconfirmed GP_1, she was not ready to completely let go of her initial idea. The existence of a locus described by $\mathrm{C}$ seemed to be a very convincing prediction for her, even though she never explicitly communicated it in these terms.

To summarize (see the visual diagram in Figure 2), at the beginning of the paperand-pencil exploration Ilaria advanced three sub-products of prediction and one more general product of GP. Looking for additional phenomenological evidence of the claim, Ilaria ran into a new prediction: gp_4, which provided an argument that was partially in contradiction to GP_1. As a consequence, another prediction was advanced for blending GP_1 and the rejection of gp_4: it was gp_5. The new insight on the configuration, provided by gp_3, gp_4, and gp_5, converged in GP_2, which was also a refined version of GP_1.

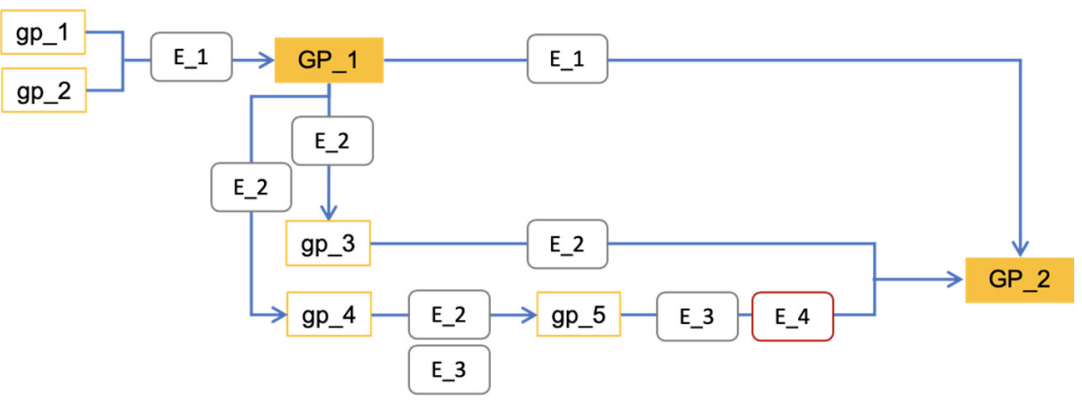

Figure 2. Visual diagram showing the connections between the products of geometric prediction (GP) and evidence advanced during the paper-and-pencil resolution of the task. 
From the beginning, Ilaria's conceptual control shaped her interaction with the drawing. Conceptual control was used for recalling a new figural concept (a parallel line through C) and reconceptualizing the figural element $C$ as a point on the line. However, such a control was not theoretical (i.e., fully Euclidean); indeed, when $\mathrm{C}$ moved along the line as Ilaria had predicted, the angle did not remain right. The spotlight showed the phenomenological evidence that Ilaria was trying to generate and allowed us to infer Ilaria's intention to investigate some discrete positions of $\mathrm{C}$ along the line. The phenomenological evidence gathered during the exploration, that seemed to be aimed at confirming her predictions, led Ilaria to refine GP_1, while firmly holding on to the underlying idea of a trajectory. Finally, she found three satisfactory (for her) positions; however, her control upon the figure was not theoretical enough to recognize that, during the prediction refinement, some theoretical constraints got lost: in each position the right angle was at $\mathrm{C}$, but $\mathrm{AB}$ was not always the hypothenuse; along the segment between two positions the angle was no longer right.

\subsubsection{Ilaria's DGE Exploration}

When the interviewer offered her the opportunity to move to the DGE, Ilaria opened the GeoGebra file that had been prepared. The DGE object was a right triangle corresponding to the figure the solver had already reasoned upon. Point $C$ can be dragged on the screen anywhere, as the right angle, by design, was softly embedded in the dynamic triangle. The measure of the angle $\mathrm{ACB}$ was also labeled; as a consequence, when $\mathrm{C}$ was moved, the label changed accordingly. Excerpt 3 was the beginning of the exploration (Table 5); the main elements captured in the analytic process are presented in Table 6.

Table 5. Excerpt 3 from Ilaria's Dynamic Geometry Environment (DGE) exploration.

\begin{tabular}{|c|c|c|}
\hline $\mathbf{N}$ & What Is Said & What Is Done \\
\hline \multirow{3}{*}{7} & \multirow{3}{*}{ Ok, ehm... } & $\begin{array}{c}\text { She starts moving } C \text { slowly on a straight trajectory, } \\
\text { parallel to AB: }\end{array}$ \\
\hline & & \\
\hline & & $\begin{array}{l}\text { Panel 6: an instance of the initial dynamic object (on the left) } \\
\text { and of the dynamic object during dragging (on the right) }\end{array}$ \\
\hline \multirow[t]{2}{*}{8} & \multirow[t]{2}{*}{ No, it changes. } & \\
\hline & & Panel 7: two instances of the dynamic object during dragging \\
\hline \multirow{3}{*}{9} & \multirow{3}{*}{ It changes. } & She changes direction, moving $C$ to the left: \\
\hline & & \\
\hline & & Panel 8: two instances of the dynamic object during dragging \\
\hline
\end{tabular}

At the beginning of the exploration, Ilaria seemed to be quite convinced that she could confirm her implicit claim C_3. To do so, she decided to move C along the predicted trajectory with the intention of reproducing the imagined discrete positions, and indeed, after initial hesitation, she quickly dragged $C$ to reach such positions. However, this DT provided contradictory evidence for her. From a cognitive point of view, this was a new approach to the task: Ilaria was only responsible for the decisions that concerned the dragging direction (possibly directed by conceptual control), the outcome of the figure transformation was managed by the software. Unlike in the paper-and-pencil environment, 
now the feedback was theoretically controlled by the software. Therefore, everything that appeared on the screen was consistent with the TEG. This allowed Ilaria to interpret such feedback as phenomenological evidence contradicting her claim. In excerpt 4 below (Table 7), we see the consequences of this; the main elements captured in the analytic process are presented in Table 8.

Table 6. Main elements from the analysis of Excerpt 3.

\begin{tabular}{|c|c|c|}
\hline Labels & Name & Description \\
\hline Spotlight & S_4 & $\begin{array}{l}\text { The spotlight is on the vertex with the } \\
\text { right angle while it is moving on the } \\
\text { parallel trajectory. AB and the triangle } \\
\text { ABC are not spotlighted. }\end{array}$ \\
\hline GP & & $\begin{array}{c}\text { No new processes are occurring; check of } \\
\text { previous GP_2, gp_3, gp_5. }\end{array}$ \\
\hline Claim & C_3 & $\begin{array}{l}\text { [C stays right as it moves along a } \\
\text { segment parallel to } A B]\end{array}$ \\
\hline \multirow{2}{*}{ Evidence } & & $\begin{array}{l}\text { DT: Ilaria intends to move } C \text { along the } \\
\text { predicted segment and she is expecting to } \\
\text { always see the right angle } \\
\left(\text { or its label marking } 90^{\circ}\right) .\end{array}$ \\
\hline & E_5 & $\begin{array}{c}\text { Phenomenological evidence: dragging C } \\
\text { on a straight trajectory, Ilaria collects } \\
\text { phenomenological evidence that } \\
\text { contradicts the claim. }\end{array}$ \\
\hline
\end{tabular}

Table 7. Excerpt 4 from Ilaria's DGE exploration.

\begin{tabular}{ll} 
What Is Said \\
\hline 10 \\
Mmm...
\end{tabular}


Table 8. Main elements from the analysis of Excerpt 4.

\begin{tabular}{|c|c|c|}
\hline Labels & Name & Description \\
\hline \multirow{3}{*}{ Spotlight } & \multirow{3}{*}{ S_5 } & $\begin{array}{l}\text { The spotlight focuses on } \mathrm{C} \text {, the label, C's } \\
\text { trajectory, to be discovered. }\end{array}$ \\
\hline & & \\
\hline & & Spotlight 4: the region of interest within the spotlight \\
\hline \multirow{2}{*}{ GP } & GP_3 & C moves along a curved trajectory. \\
\hline & gp_6 & C moves on a circle. \\
\hline Claim & $C_{-4}$ & [When the angle is right] C moves on a circle. \\
\hline \multirow[b]{2}{*}{ Evidence } & & $\begin{array}{l}\text { MD: Ilaria tries to maintain both the right angle } \\
\text { and some kinds of trajectories that, a priori, } \\
\text { are unknown to her. }\end{array}$ \\
\hline & E_6 & $\begin{array}{l}\text { Phenomenological evidence (which is consistent } \\
\text { with the TEG because controlled by the DGE) } \\
\text { allows the solver to find the claim that arises } \\
\text { during the generation of evidence. }\end{array}$ \\
\hline
\end{tabular}

Since Ilaria's predictions were disconfirmed by the DGE's feedback, she decided to explore the dynamic figure in a different way: she first seemed to be searching for possible positions in which C's label would mark $90^{\circ}$, then she initiated a constructive dialogue with the figure moving $C$ on small arcs of what she eventually conceived as a circle. Ilaria's prediction about the circle seemed to be constructed progressively. Eventually, she used MD and stated that $C$ should describe a circle. Among the loci that she knew, Ilaria seemed to choose the circle as the most suitable or the only one at her disposal. This depended on her conceptual control and development of figural concepts of curves.

Ilaria's exploration seemed to be driven by a general prediction of the figure: from a phenomenological point of view she expected to find a trajectory; from the theoretical point of view, this expectation was settled in the solver's idea that there must exist a locus for C. The circle was the result of a constructive combination between the continuous motion of $C$ under MD, the focus on a certain trajectory, and the DGE feedback. Apparently, it was the fact that Ilaria was looking for a trajectory that led her to the correct locus for $\mathrm{C}$. We could say that Ilaria was "ready" to see the locus: this readiness seemed to be heavily rooted in her conceptual control, which allowed her to notice the regularity.

Moreover, the DGE feedback supported Ilaria in this process by allowing her to focus on $C$ and observe its behavior, while being sure that the other properties ( $A B$ fixed, $A B C$ triangle) were coherently maintained. Since the feedback is always theoretically controlled by the rules embedded into the software, the solver can pay more attention to the discovery of unpredicted invariants.

After the interviewer asked Ilaria to better explain the last utterance, excerpt 5 started (Table 9); the main elements captured in the analytic process are presented in Table 10. 
Table 9. Excerpt 5 from Ilaria's interview.

\begin{tabular}{|c|c|c|}
\hline $\mathbf{N}$ & What Is Said & What Is Done \\
\hline 13 & $\begin{array}{l}\text { Like...if I try...come on, let's see if I } \\
\text { can get it the right size. } \\
90,90,90 \ldots 100 .\end{array}$ & $\begin{array}{l}\text { She drags } C \text { fast on the right. Then } \\
\text { she moves } C \text { slowly, following a } \\
\text { curvilinear trajectory. }\end{array}$ \\
\hline 14 & $\begin{array}{l}\text { Olpà! I'm at } 90 . \text { If I, like I move it in a } \\
\text { circle, it stays more or less...or } \\
\text { less...no! }\end{array}$ & $\begin{array}{l}\text { Panel 12: configuration at which Ilaria } \\
\text { stops dragging } \\
\text { She slowly moves C on a very small } \\
\text { portion of a circle and stops when the } \\
\text { label is no longer " } 90^{\circ} \text { ". }\end{array}$ \\
\hline 15 & I have no idea. I lost all hope. Well. & $\begin{array}{l}\text { She moves } C \text { to a position where she } \\
\text { knows the angle will be right. }\end{array}$ \\
\hline
\end{tabular}

Table 10. Main elements from the analysis of Excerpt 5.

\begin{tabular}{|c|c|c|}
\hline Labels & Name & Description \\
\hline Spotlight & S_6 & $\begin{array}{l}\text { The spotlight is on the vertex } C \text { and the label } \\
\text { with the measure. The spotlight moves as } \\
\text { C is dragged. }\end{array}$ \\
\hline GP & & $\begin{array}{l}\text { No new processes are occurring; } \\
\text { Ilaria checks previous } g p \_6 \text {. }\end{array}$ \\
\hline Claim & C_5 & When $\mathrm{C}$ is on a circle [the angle is right]. \\
\hline \multirow{3}{*}{ Evidence } & & $\begin{array}{l}\text { MD (utt. 13): the solver explicitly states her } \\
\text { intention of moving C so that the angle is } \\
\text { right or the label is " } 90^{\circ} \text { ". }\end{array}$ \\
\hline & & $\begin{array}{l}\text { MD (utt. 14): the solver moves C on a circle. } \\
\text { DT (utt. 14): the solver tests if, moving C on } \\
\text { a small arc of a circle, the angle is right. }\end{array}$ \\
\hline & E_7 & $\begin{array}{l}\text { Phenomenological evidence: dragging } \mathrm{C} \text { on a } \\
\text { circle, Ilaria expects to always see the right } \\
\text { angle (or its label)_this feedback provides } \\
\text { her with phenomenological } \\
\text { evidence for her claim. }\end{array}$ \\
\hline
\end{tabular}

Ilaria's intention changed during the exploration. At the beginning, Ilaria was looking for angles that measured $90^{\circ}$ (utt. 13); to do so, she dragged C following a curvilinear path, along which she expected to find such angles. This was MD. However, she lost the right angle ("100", utt.13). Then she found another suitable configuration (see "Olpà! I'm at 90" utt. 14) and stated her intention of moving $C$ along small arcs of a circle, and she dragged $\mathrm{C}$ accordingly. Now DT was used to check if, while moving $\mathrm{C}$ along small arcs of a circle, the angle actually stayed right.

These two manipulations were different in terms of the relationship between the locus of $C$ and the right angles. During the first, the focus was on the angle measures; following the circle was only a way to obtain right angles. We interpreted this as an attempt to generate phenomenological evidence for the claim $C_{-} 4$, as if Ilaria were asking the software for instances of right angles. During the second manipulation, Ilaria's focus was on the 
trajectory: the labels were the way she could check the coherence of the manipulation. We interpreted this as an attempt to generate phenomenological evidence for the claim C_5.

The two manipulations were similar in terms of their relationship with the claim. In both cases the claim had a premise and a conclusion; during the DGE exploration, Ilaria seemed to only control directly the property in the premise (C moves in a circle), and she seemed to be asking the software to produce the property in the conclusion (the angle is right). Ilaria needed to use conceptual control to interpret the feedback as actually supporting the conclusion, and then to decide to change, confirm, or reject the claim.

However, since she had not obtained as many instances of right angles as she seemed to want (utt. 15), Ilaria's interpretation of the DT was that it failed, and she declared to have no idea about how to continue. The focus of the spotlight did not seem to be convincing enough to confirm her prediction. Moreover, she seemed to trust in the DGE's feedback more than her prediction, to the extent that when she saw just a few non-right angles, she was ready to completely reject her prediction ("I lost all hope" utt. 15).

Since Ilaria seemed to be stuck, the interviewer asked how she was moving $C$ during the last exploration. Below is her answer (Table 11); the main elements captured in the analytic process are presented in Table 12.

Table 11. Excerpt 6 from Ilaria's DGE exploration.

She moves the pointer on the screen, mimicking a circle:

Eh like in a circle. In a circle, like an arc of a circle.

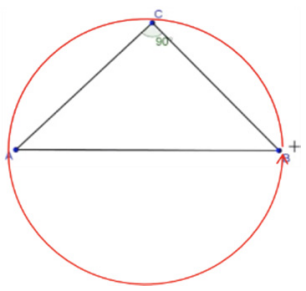

Panel 13: screen capture of the configuration with a red arrow (added by us) reproducing the pointer's movement

Then, she mimics twice a semicircle:

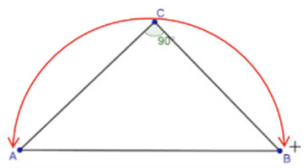

Panel 14: screen capture of the configuration with a red arrow reproducing the pointer's movement

Because I remembered something else.

She looks at the drawing on the sheet of paper.

She moves the pen on the drawing and mimics a semicircle through B, C, and A:

That basically...with respect to a

right angle it's...A and B is always...the diameter.

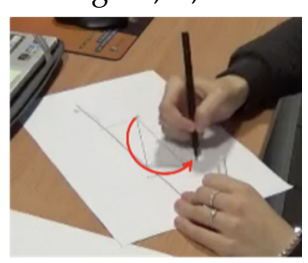

Panel 15: Ilaria's gesture on the drawing

She adds a circle on the drawing: draw a circle.

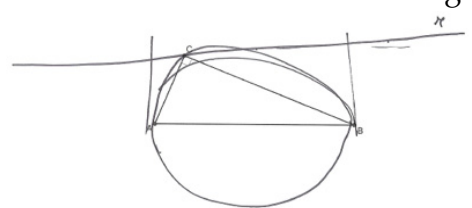

Panel 16: a snapshot of the new drawing 
Table 11. Cont.

What Is Said
No $\begin{gathered}\text { So, theoretically if I move C } \\ \text { along the..the arc of the circle, it } \\ \text { should come out...ehm...it } \\ \text { should still be a right triangle. }\end{gathered}$
Panel 17: Ilaria's gestures on the new drawing

Table 12. Main elements from the analysis of Excerpt 6.

\begin{tabular}{|c|c|c|}
\hline Labels & Name & Description \\
\hline Spotlight & S_5 & $\begin{array}{l}\text { The spotlight contains: the whole triangle, and in particular } \\
\text { AB; the circumscribed circle or part of it (the semicircle). } \\
\text { The segment AB in the spotlight has a twofold } \\
\text { conceptualization: it is the hypotenuse of the triangle and a } \\
\text { diameter of the circle. }\end{array}$ \\
\hline GP & $\begin{array}{l}\text { gp_7 } \\
\text { gp_8 } \\
\text { gp_9 }\end{array}$ & $\begin{array}{l}\text { a circle around the triangle } \\
\text { a semicircle around the triangle } \\
\text { a circle with diameter } A B\end{array}$ \\
\hline Claim & C_6 & [C moves on a] a circle with diameter $A B$ \\
\hline \multirow[t]{2}{*}{ Evidence } & E_8 & $\begin{array}{c}\text { Phenomenological evidence: mimicking and drawing the } \\
\text { circle and the semicircle, Ilaria provides phenomenological } \\
\text { evidence to the claim, by making explicit her prediction } \\
\text { about the circle. }\end{array}$ \\
\hline & E_9 & $\begin{array}{l}\text { Theoretical evidence: the fragment of theory concerning the } \\
\text { circle that can be circumscribed to a right triangle provides a } \\
\text { theoretical evidence to the claim and predictions. }\end{array}$ \\
\hline
\end{tabular}

The excerpt shows the generation of evidence and predictions that were strongly influenced by the DGE exploration. First, Ilaria recalled her last prediction on C (gp_6), but her gesture suggested that she added new information to her prediction: the circle that she is mimicking enclosed the whole triangle passing through its vertices (gp_7). This product of prediction constituted a refined version of gp_6. Such a gesture supported the production of phenomenological evidence of the claim C_6, by depicting the imagined circle. The theoretical evidence was more and more integrated into Ilaria's processes. For the first time, now Ilaria referred to the circle using the proper mathematical term, which focused specifically on the border; indeed, in Italian we use two different terms for referring to the portion of the plane within a circle and to the border of the circle. Then she reported on an attempt to recall a mathematical theorem that she knew ("I remembered" utt. 17): a right triangle is always inscribable into a circle that has the hypotenuse as a diameter. Even if she did not formally recall the complete theorem, this was what Ilaria's utterances and gestures seemed to suggest. In particular, Ilaria stressed the generality of her statement: AB is "always" a diameter of the mimicked circle. This consideration seemed to provide Ilaria with theoretical evidence for the claim and it led her to refining her prediction. 
Ilaria's attempt to collect phenomenological evidence and describe the outcome to the interviewer seemed to solicit her conceptual control, which led to her recalling a suitable fragment of theory (the theorem she stated in utt. 18). Now the spotlight on the dynamic figure needed to be enlarged in order to also include the theoretical evidence provided by the theorem: the spotlight on the drawing now shone on $\mathrm{AB}$ and the whole triangle. More interestingly, as an effect of Ilaria's conceptual control, $A B$ acquired a twofold theoretical status: $\mathrm{AB}$ was the hypotenuse of the triangle, but at the same time $\mathrm{AB}$ was the diameter of the circle. Indeed, this is one of the functions of conceptual control: seeing the same figural element as part of different figural concepts [48]. Supported by the DGE exploration, Ilaria now activated a conceptual control that was more versatile and consistent with the TEG.

Drawing the circle on the sheet of paper (see Panel 15), Ilaria searched for additional phenomenological evidence for the claim, and she stated the prediction explicitly. Once the circle was drawn out, the implicit claim was revealed, and it became explicit: the several processes of GP accomplished by Ilaria contributed to a more general process of conjecture-generation. Moreover, at the end of the DGE exploration, when the interviewer repeated the very first question: "What can you say about the vertex with the right angle?", Ilaria said: "That if I want it to remain right [...] I would have to move it along an imaginary circle with diameter $A B . "$ This utterance expressed another and quite detailed idea of the solution.

To summarize, the DGE exploration changed Ilaria's conceptualization of the objects in focus. In particular, the DGE feedback supported conceptual control, allowing Ilaria to recall new theoretical elements that were coherent with the TEG. In return, the introduction of new theoretical elements that were inspired by the DGE feedback empowered Ilaria's conceptual control, leading it to become more theoretical.

With respect to prediction, during the DGE exploration, the interplay between GPs can be summarized as follows (see also Figure 3). A very general prediction drove Ilaria's interaction with the dynamic figure (GP_3). As in the paper-and-pencil environment, she was quite convinced that point $C$ must follow a trajectory: all of Ilaria's attempts to manipulate the figure were devoted to finding the most suitable path. First of all, she tested the previously predicted line and some specific positions on that trajectory. Even if the DGE feedback revealed a mismatch between Ilaria's figural expectations and the behavior of the dynamic figure under dragging, she seemed to always keep GP_3 in the background of her reasoning. Indeed, right after GP_2 was disconfirmed by the DGE feedback, she started moving $\mathrm{C}$, looking for small pieces of a round trajectory along which the angle was right. Once she observed as many instances of right angles as she needed, Ilaria communicated her prediction gp_6. As she started to drag, she had not yet communicated a detailed prediction; she seemed to be asking the dynamic figure for information about the trajectory she was looking for, and thus to help her make a prediction. In this sense, the circle was a prediction that partially stemmed from the exploration, and, thus, was not a genuine prediction. The visual diagram below (Figure 3) shows how, starting from a very general prediction (GP_3) and supported by the DGE feedback, Ilaria refined her predicted trajectory more and more by adding theoretical details (i.e., type of locus and principal information to reproduce).

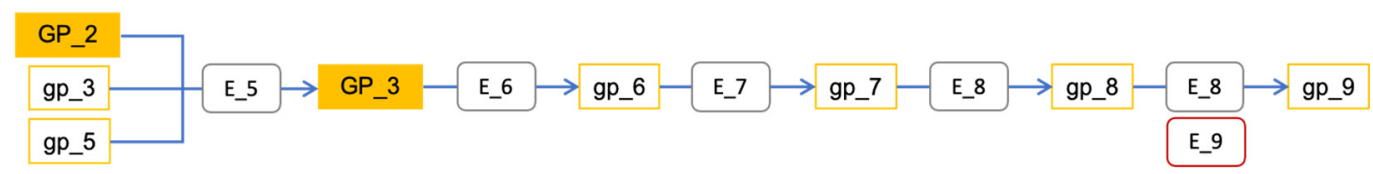

Figure 3. Visual diagram showing connections between the products of GP and evidence advanced during Ilaria's DGE exploration.

The dragging modalities Ilaria used to interact with the dynamic figure highlighted a cyclic approach: she wanted to see a trajectory; she asked the dynamic figure for a prediction while she was dragging $C$, trying to maintain the right angle; she observed the effect of the manipulation and inferred the prediction; she tested the product of GP, while $\mathrm{C}$ was dragged accordingly; finally, based on the feedback provided by the software, she 
adjusted the prediction. During the continuous manipulation of $C$, these steps can happen so fast that the observations of the solver's intention and of the outcome overlap.

\section{Discussion and Conclusions}

The study presented in this paper is explorative, and the findings provide new insight into geometric prediction, a very specific cognitive process. The study presents the case of a 9th-grade high school student solving a prediction open problem, first within a paperand-pencil environment, and then within a DGE. The fine-grained qualitative analyses provide a richness in detail and depth that would not have been reachable through a different methodology. The findings presented above support the claim that, during the resolution of prediction open problems, processes of GP are strongly influenced by the solver's conceptual control; moreover, the findings support the hypothesis that the use of DGEs can be a catalyst for the evolution of the solver's conceptual control toward a control that is closer to theoretical control. The analyses also lead us to new theoretical and educational hypotheses on the possible evolution of a student's conceptual control.

In this study we found that the solver's conceptual control over the figure influenced the whole resolution process. The most evident instances of conceptual control were related to Ilaria's persistent underlying idea that $C$ must describe a continuous trajectory (i.e., a line, a segment, a circle, a semicircle) and to her recalling of a known mathematical theorem. We observed how Ilaria's initial conceptualization of the problem influenced her continuous approach: she was thinking in terms of looking for a suitable path for $\mathrm{C}$ in order to maintain all the given theoretical constraints. This first interpretation became the leitmotif that shaped all of Ilaria's GP processes. Even when figural elements seen within the drawing or the DGE feedback disconfirmed her predictions, Ilaria did not change her intention of finding a trajectory. This was evident within both the paper-and-pencil environment and the DGE. In the paper-and-pencil environment, Ilaria predicted a first trajectory that was modified according to the phenomenological evidence provided by new information, but its existence was never questioned. In the DGE, when GP_2 was disconfirmed by the feedback, very small curvilinear trajectories were enough for Ilaria to predict an entire circle.

Ilaria's conceptualization of the figural concept of locus also played a prominent role. Indeed, among all the possible loci, she focused only on lines and circles. For example, a priori there were no reasons for excluding an ellipse; however, the figural concept of ellipse, in terms of a locus of points that satisfies a certain rule, did not seem to be experienced by Ilaria at the time of the interview; instead, we know that circles and parallel lines were well-known figural concepts for her. So, lines and circles (or some part of them) were the only possible trajectories at her disposal.

The outcome of the first process of GP was a line parallel to AB. Once the line was shown through gestures, the solver tried to recall a suitable mathematical result that might support her prediction (utt. 2). Her productions suggested that Ilaria was trying to recall a fragment of theory: the area of the triangle is an invariant under the translation of a vertex along a line parallel to the opposite side. This implicit theoretical evidence provided by the theorem was not coherent with the given constraints (the right angle was lost), albeit the underlying theorem was consistent in itself. So, we can conclude that Ilaria's conceptual control was not sufficiently coherent with the TEG for corroborating or rejecting GP_1 (see also "If I'm not mistaken").

Within the paper-and-pencil environment manipulating figural elements under conceptual control can be quite challenging for the solvers [14]. Although Ilaria's utterances (see, for example, "moving along this line," "remains always right") revealed that she was reasoning in terms of a trajectory rather than discrete positions for $\mathrm{C}$, she adopted a discrete approach in an attempt to produce phenomenological evidence. In the absence of convincing theoretical evidence, Ilaria needed to fix some snapshots of the figure as she imagines it to continuously move $\mathrm{C}$ along the line to confirm or disconfirm her predictions. Starting from the position of $\mathrm{C}$ she pointed to, she had to imagine and reconstruct the 
corresponding angle. In other words, she had to control not only the position of $C$ but also the outcome of the movement. In order to manage all these aspects while checking the consistency with the TEG, the solver needs a very strong conceptual control. In the paper-and-pencil environment, we interpreted Ilaria's success in terms of her ability to manage the necessary cognitive load "by compartmentalising the representation of the geometry from its manipulation, alleviating the need to store multiple instances of the model in the working memory" [49] (p. 359).

Instead, in the DGE, the phenomenological outcome of the manipulation of a point on the screen is provided to the solver by the software. In this sense, paraphrasing and applying some of Buckley and colleagues' findings [49] to our context, we can say that having a DGE at the solver's disposal has the effect of removing the need to retain the figure "in the working memory allowing more resources to be allocated for other pertinent cognitive actions specific to the problem-solving approach being adopted" [49] (p. 358). Moreover, the theoretical consistency of the outcome is assured by the rules of the TEG embedded into the software. The solver only has to interpret the outcome using conceptual control. In this view, the solver's conceptual control is shared with the DGE, which contributes exercising theoretical control over the figure. Being sure of the consistency of the outcome, the solver can use conceptual control for producing new theoretical evidence rather than for controlling the coherence of the manipulation.

At the beginning of the DGE exploration, the mismatch between what the solver is expecting to see and what actually is on the screen suggests that her conceptual control is far from the sought-after theoretical control. However, passing through a constructive interplay between MD and DT, the DGE exploration guides the solver to recognize figural components of a circle. This predicted circle triggers a reconceptualization of the figure that is seen as a right triangle constructed upon the given diameter of a circle. This is possible because the solver recalls a specific mathematical result. The result was not suggested by the interviewer, but it was part of Ilaria's TEG. The feedback provided by the DGE during exploration only solicited this specific fragment of TEG that the solver had at her disposal but did not activate during the paper-and-pencil session. Seemingly, the dynamic exploration progressively changed Ilaria's conceptual control, turning it into theoretical control, that is, a conceptual control that is more versatile and consistent with the TEG.

Moreover, our findings suggest that the different nature of the interactions with drawings or DGE objects might affect the solver's conceptualization of the figure. Analyses suggest that the reason lies right at the heart of the software design: movements and identification of invariants. When a point of a figure is dragged, the software provides a sequence of images based on the movement of the point. These images show many instances of the figure that can be perceived as the "same object" under transformation [15-50]. The fact is that it is impossible to move a point on the screen from one position to another without displaying all the intermediate positions: each position immediately generates a new figural instance of the rest of the figure. Solvers can decide whichever of the intermediate objects to focus on (or not), but, in any case, these are available on the screen at any time during dragging. Moreover, when a solver drags a point, the figural feedback is immediately generated by the DGE: input and output are simultaneous. As a consequence, the solver can change or form her conceptualization of the figure during the transformation due to the phenomenological feedback. In this sense, the figural components supported by the image on the screen have the opportunity to progressively change and shape the conceptual components. This is not possible within the paper-and-pencil environment unless the solver has a very strong conceptual control that supports imagined continuous transformations. Indeed, Ilaria preferred to transform the triangle discretely and observe if the figural outcome was consistent with her conceptualization.

From an educational point of view, this finding suggests that actually DGEs can be useful environments for adapting the POE sequence to geometrical reasoning in the domain of Euclidean Geometry. The possibility of perceiving dynamic objects as geometrical figures in motion within a DGE provides solvers with a context to figurally test their prediction. 
However, the interaction with a dynamic figure cannot be totally assimilated to a physical experiment. As stressed before and highlighted in our analyses, the reason why this is a delicate matter relies also on the nature of geometrical objects. Indeed, Ilaria's interview showed how theoretical elements play a crucial role in generating, testing, and refining predictions. Moreover, a certain amount of conceptual control is needed to see a DGE object as a dynamic figure and to interpret the feedback as evidence of a certain claim. For example, in some cases, solvers are not able to catch the mismatch between their previously communicated expectations and the DGE's feedback [5]. Such a mismatch is only evident for the interviewer. Furthermore, our analyses reveal how, since the very beginning, Ilaria made use of a certain conceptual control that guided her interactions with the figure. However, some questions still remain open concerning cases in which a solver is not able to constructively interpret the DGE's feedback. In light of this study, an emerging hypothesis is that a conceptual control that was not previously constructed upon adequate geometrical experiences can affect dynamic explorations. In this case, it can be quite interesting to investigate students' difficulties in geometry and explore both the possibilities and the shortcomings of transposing the POE sequence into the domain of geometrical reasoning. In order to further address this latter issue, some epistemic and ontological studies are necessary.

Our findings are consistent with previous studies on prediction. Following Kasmer and Kim [3] (p. 188): "posing prediction questions to students prior to exploring the mathematical ideas of a problem creates learning opportunities for students, by encouraging them to evoke prior knowledge and to bridge between mathematical concepts (e.g., previous concepts and a novel one)." As well as for prediction in a broader sense, at the end of a GP process the solver has gained new insights into the geometric figure $s /$ he has manipulated, transforming the initial geometric configuration to produce a new configuration. Therefore, a product of GP is actually a new geometrical object for the solver, that is, a figural concept with newly discovered figural and conceptual components. There is more. Findings suggest that such a "bridge" between figural concepts is accessible thanks to the solver's possibility to establish connections between and recall suitable figural concepts, to generate and recognize evidence, to look at the same figural element as part of several figural concepts, just to list a few. All of these mental acts are supported by the solver's conceptual control. In short, the learning opportunities rely on the possibility to stimulate and eventually modify the solver's conceptual control. This study has provided new analytical tools for studying such a process, and we hope that they will be used in future research in this domain. Indeed, our framework combines two lenses: the Theory of Figural Concepts [27] and the theoretical construct of evidence [31], allowing us to construct an analytical procedure useful for gathering information on a complex construct, which conceptual control is. Further studies on this topic can benefit from our attempt.

Our findings are in line with previous studies on anticipations within the DGE exploration. Hollebrands looks at the mutual influence of predictions and DGE exploration by distinguishing between the solver's use of proactive or reactive strategies [16]; what makes the difference between the two strategies is, respectively, the identification of presence or absence of a "plan in mind" that is strictly related to the ability to anticipate. We have observed how Ilaria's previously communicated predictions affected her proactive interaction with the dynamic object. Moreover, the use of proactive strategies is considered to be guided by the solver's "understanding of geometrical properties and relations" [16] (p. 184) that in our framework is supported by conceptual control. Our findings are in line with this claim, but there is more. The software produces feedback even when the solvers proactively interact with a dynamic figure, that is, when the solvers move objects according to a certain prediction; in return, such feedback might influence, change or refine solvers' predictions as far as they are willing to change their conceptualization of the figure that depends on their conceptual control. So, during the DGE exploration many cycles of interpretation of feedbacks and refinement of predictions arise; later, such predictions could affect a new proactive strategy. The study of these cycles can be a new fruitful line 
of research. Finally, since Hollebrands highlighted that encouraging the use of proactive strategies among the students is a challenging task for teachers [16], we believe that an explicit request for predictions, before the interaction with a DGE, might trigger a more natural transition from reactive to proactive strategies.

From a cognitive point of view, this study contributes to the research on visuo-spatial abilities by stressing the fundamental role of and intertwined relationship with elements that belong to the solver's knowledge and conceptualization of geometry. As suggested by other researchers [22], there is still the necessity to consider new theoretical perspectives to take into account the influence of specific knowledge on these abilities.

As regards prediction, our findings suggest that the use of prediction open problems such as those developed for this study could strengthen a student's skill of geometric prediction, since his/her conceptual control can be progressively modified by the DGE's feedback, becoming more theoretical. In this sense, if our findings were confirmed by further qualitative and quantitative research in this direction, they could inform educational practices. On the one hand, geometrical activities are effective when they support the harmonization between the figural and the conceptual components in a figural concept; on the other hand, the activities need to promote conceptual development that is consistent with the reference theory, and, consequently, to stimulate students' conceptual control on figural elements to become more and more theoretical. A careful design and implementation of geometrical activities that make use of prediction open problems coupled with a DGE exploration might help in this respect.

There is still much to learn on geometric prediction and on the actual benefits of its routine use within classroom geometrical activities; however, this study represents a step forward in this direction.

Author Contributions: Conceptualization, E.M.; methodology, E.M.; formal analysis, E.M.; investigation, E.M.; writing—original draft preparation, E.M.; writing—review and editing, E.M. and A.E.B.-F.; visualization, E.M.; supervision, A.E.B.-F. All authors have read and agreed to the published version of the manuscript.

Funding: This research was funded by a PhD fellowship in Mathematics awarded to Elisa Miragliotta by the University of Modena and Reggio Emilia.

Institutional Review Board Statement: The study was conducted according to the guidelines of the Declaration of Helsinki, and approved by the University of Ferrara (protocol code 1026-30 November 2017).

Informed Consent Statement: Informed consent was obtained from all subjects involved in the study.

Data Availability Statement: Other data from interviews conducted during the doctoral work of the first author are available at https: / / drive.google.com/open?id=11tiLfw8b33R2vUGnsXccUabsEZzrM7 $\mathrm{cE}$ and analyzed in her dissertation [6].

Acknowledgments: We wish to thank Maria Alessandra Mariotti, with whom we have discussed at length Fischbein's Theory of Figural Concepts. Discussions and exchanges with her led to many ideas in this paper.

Conflicts of Interest: The authors declare no conflict of interest.

\section{References}

1. White, R.; Gunstone, R. Probing Understanding; Routledge: London, UK, 2014. [CrossRef]

2. Lim, K.H.; Buendia, G.; Kim, O.K.; Cordero, F.; Kasmer, L. The role of prediction in the teaching and learning of mathematics. Int. J. Math. Educ. Sci. Technol. 2010, 41, 595-608. [CrossRef]

3. Kasmer, L.A.; Kim, O.K. The nature of student predictions and learning opportunities in middle school algebra. Educ. Stud. Math. 2012, 79, 175-191. [CrossRef]

4. Mariotti, M.A.; Baccaglini-Frank, A. Developing geometrical exploration skills through dynamic geometry. In Broadening the Scope of Research on Mathematical Problem Solving: A Focus on Technology, Creativity and Affect; Carreira, S., Amado, N., Jones, K., Eds.; Springer: Cham, Germany, 2018; pp. 153-176. [CrossRef] 
5. Miragliotta, E.; Baccaglini-Frank, A. You see (mostly) what you predict: The power of geometric prediction. In Proceedings of the 42nd Conference of the International Group for the Psychology of Mathematics Education, Umeå, Sweden, 3-8 July 2018; Bergqvist, E., Österholm, M., Granberg, C., Sumpter, L., Eds.; PME: Umeå, Sweden, 2018; Volume 3, pp. 387-394.

6. Miragliotta, E. Geometric Prediction: A Model to Analyze a Cognitive Process in Geometrical Problem-Solving. Ph.D. Thesis, University of Modena and Reggio Emilia, Reggio Emilia, Italy, 2020. Available online: http://hdl.handle.net/11380/1200566 (accessed on 22 March 2021).

7. Kim, O.K.; Kasmer, L. Using Prediction to Promote Mathematical Reasoning. Math. Teach. Middle Sch. 2007, 12, 294-299. [CrossRef]

8. Mariotti, M.A. Transforming Images in a DGS: The semiotic potential of the dragging tool for introducing the notion of conditional statement. In Transformation-A Fundamental Idea of Mathematics Education; Rezat, S., Hattermann, M., Peter-Koop, A., Eds.; Springer: New York, NY, USA, 2015; pp. 155-172. [CrossRef]

9. Sinclair, N.; Robutti, O. Technology and the role of proof: The case of dynamic geometry. In Third International Handbook of Mathematics Education; Clements, M.A.K., Bishop, A., Keitel-Kreidt, C., Kilpatrick, J., Leung, F.K.-S., Eds.; Springer: New York, NY, USA, 2013; pp. 571-596.

10. Baccaglini-Frank, A.; Antonini, S.; Leung, A.; Mariotti, M.A. From pseudo-objects in dynamic explorations to proof by contradiction. Dig. Exp. Math. Educ. 2018. [CrossRef]

11. Laborde, J.M.; Strässer, R. Cabri-Géomètre: A microworld of geometry for guided discovery learning. Zentralblatt Für Didaktik Der Mathematic 1990, 90, 171-177.

12. Hadas, N.; Hershkowitz, R.; Schwarz, B.B. The Role of Contradiction and Uncertainty in Promoting the Need to Prove in Dynamic Geometry Environments. Educ. Stud. Math. 2000, 44, 127-150. [CrossRef]

13. Mariotti, M.A. Introduction to Proof: The Mediation of a Dynamic Software Environment. Educ. Stud. Math. 2000, 44, 25-53. [CrossRef]

14. Baccaglini-Frank, A. Conjecturing in Dynamic Geometry: A Model for Conjecture-Generation through Maintaining Dragging. Ph.D. Thesis, University of New Hampshire, Durham, NH, USA, 2010. Available online: https://scholars.unh.edu/dissertation/ 529 (accessed on 22 March 2021).

15. Leung, A.; Baccaglini-Frank, A.; Mariotti, M.A. Discernment in dynamic geometry environments. Educ. Stud. Math. 2013, 84, 439-460. [CrossRef]

16. Hollebrands, K.F. The role of a dynamic software program for geometry in the strategies high school mathematics students employ. J. Res. Math. Educ. 2007, 38, 164-192.

17. Bruce, C.D.; Davis, B.; Sinclair, N.; McGarvey, L.; Hallowell, D.; Drefs, M.; Francis, K.; Hawes, Z.; Moss, J.; Mulligan, J.; et al. Understanding gaps in research networks: Using "spatial reasoning" as a window into the importance of networked educational research. Educ. Stud. Math. 2017, 95, 143-161. [CrossRef]

18. Miragliotta, E.; Baccaglini-Frank, A. Visuo-spatial abilities and geometry: A first proposal of a theoretical framework for interpreting processes of visualization. In Proceedings of the Tenth Congress of the European Society for Research in Mathematics Education CERME10, Dublin, Ireland, 1-5 February 2017; Dooley, T., Gueudet, G., Eds.; DCU Institute of Education and ERME: Dublin, Ireland, 2017; pp. 3952-3959.

19. Cornoldi, C.; Vecchi, T. Visuo-Spatial Working Memory and Individual Differences; Psychology Press: Hove, UK, 2003.

20. Giofrè, D.; Mammarella, I.C.; Ronconi, L.; Cornoldi, C. Visuospatial working memory in intuitive geometry, and in academic achievement in geometry. Learn. Individ. Differ. 2013, 23, 114-122. [CrossRef]

21. Mammarella, I.C.; Giofrè, D.; Ferrara, R.; Cornoldi, C. Intuitive geometry and visuospatial working memory in children showing symptoms of nonverbal learning disabilities. Child Neuropsychol. 2013, 19, 235-249. [CrossRef] [PubMed]

22. Buckley, J.; Seery, N.; Canty, D. A heuristic framework of spatial ability: A review and synthesis of spatial factor literature to support its translation into STEM education. Educ. Psychol. Rev. 2018, 30, 947-972. [CrossRef]

23. Sinclair, N.; Bartolini Bussi, M.G.B.; de Villiers, M.; Jones, K.; Kortenkamp, U.; Leung, A.; Owens, K. Recent research on geometry education: An ICME-13 survey team report. ZDM 2016, 48, 691-719. [CrossRef]

24. Hershkowitz, R.; Ben-Chaim, D.; Hoyles, C.; Lappan, G.; Mitchelmore, M.; Vinner, S. Psychological aspects of learning geometry. In Mathematics and Cognition, ICMI Study Series; Nesher, P., Kilpatrick, J., Eds.; Cambridge University Press: Cambridge, UK, 1989; pp. 70-95. [CrossRef]

25. Vinner, S. The role of definitions in the teaching and learning of mathematics. In Advanced Mathematical Thinking; Tall, D.O., Ed.; Kluwer Academic Publishers: Dordrecht, The Netherlands, 1991; pp. 65-81. [CrossRef]

26. Clements, D.H.; Battista, M.T. Geometry and spatial reasoning. In Handbook of Research on Mathematics Teaching and Learning; Grouws, D.A., Ed.; Macmillan Pub. Co.: New York, NY, USA, 1992.

27. Fischbein, E. The theory of figural concepts. Educ. Stud. Math. 1993, 24, 139-162. [CrossRef]

28. Mariotti, M.A. Geometrical reasoning as a dialectic between the figural and the conceptual aspects. Struct. Topol. 1992, 18, 9-18.

29. Lopez-Real, F.; Leung, A. Dragging as a conceptual tool in dynamic geometry environments. Int. J. Math. Educ. Sci. Technol. Technol. 2006, 37, 665-679. [CrossRef]

30. Laborde, C. The Hidden Role of Diagrams in Students' Construction of Meaning in Geometry. In Meaning in Mathematics Education; Kilpatrick, J., Hoyles, C., Skovsmose, O., Valero, P., Eds.; Springer: New York, NY, USA, 2005; Volume 37, pp. 159-179. [CrossRef] 
31. Baccaglini-Frank, A. Dragging, instrumented abduction and evidence, in processes of conjecture generation in a dynamic geometry environment. ZDM 2019, 51, 779-791. [CrossRef]

32. Ginsburg, H. The clinical interview in psychological research on mathematical thinking: Aims, rationales, techniques. Learn. Math. 1981, 1, 4-11.

33. Goldin, G. A scientific perspective on structures, task-based interviews in mathematics education research. In Research Design in Mathematics and Science Education; Lesh, R., Kelly, A.E., Eds.; Lawrence Erlbaum Associates: Hillsdale, MI, USA, 2000; pp. 517-545.

34. diSessa, A.A. An interactional analysis of clinical interviewing. Cogn. Instr. 2007, 25, 523-565. [CrossRef]

35. Baxter, P.; Jack, S. Qualitative case study methodology: Study design and implementation for novice researchers. Qual. Rep. 2008, 13, 544-559. [CrossRef]

36. MIUR. Indicazioni Nazionali per il Curricolo Della Scuola Dell'infanzia e del Primo Ciclo D'istruzione. Available online: http: //www.indicazioninazionali.it/wp-content/uploads/2018/08/Indicazioni_Annali_Definitivo.pdf (accessed on 22 March 2021).

37. Arsac, G.; Germain, G.; Mante, M. Problème Ouvert et Situation-Problème; IREM: Villeurbanne, France, 1988.

38. Silver, E.A. The Nature and Use of Open Problems in Mathematics Education: Mathematical and Pedagogical Perspectives. ZDM/Int. Rev. Math. Educ. 1995, 27, 67-72.

39. Mogetta, C.; Olivero, F.; Jones, K. Providing the Motivation to Prove in a Dynamic Geometry Environment. In Proceedings of the British Society for Research into Learning Mathematics, Lancaster, UK, 5 June 1999; Bills, L., Ed.; St Martin's University College: Lancaster, UK, 1999; Volume 19, pp. 91-96.

40. Mariotti, M.A.; Bartolini Bussi, M.; Boero, P.; Ferri, F.; Garuti, R. Approaching geometry theorems in contexts: From history and epistemology to cognition. In Proceedings of the 21th PME Conference; PME: Lathi, Finland, 1997; Volume 1, pp. $180-195$.

41. Healy, L. Identifying and explaining geometric relationship: Interactions with robust and soft Cabri constructions. In Proceedings of the 24th Conference of the IGPME, Hiroshima, Japan, 23-27 July 2000; Volume 1, pp. 103-117.

42. Holzl, R. How does dragging affect the learning of geometry? Int. J. Comput. Math. Learn. 1996, 1, 169-187. [CrossRef]

43. Arzarello, F.; Olivero, F.; Paola, D.; Robutti, O. A cognitive analysis of dragging practices in Cabri environments. ZDM 2002, 34, 66-72. [CrossRef]

44. Healy, L.; Hoyles, C. Software tools for geometrical problem solving: Potentials and pitfalls. Int. J. Comput. Math. Learn. 2001, 6, 235-256. [CrossRef]

45. Baccaglini-Frank, A.; Mariotti, M.; Antonini, S. Different perceptions of invariants and generality of proof in dynamic geometry. In Proceedings of the 33rd Conference of the International Group for the Psychology of Matematics Education, Thessaloniki, Greece, 19-24 July 2009; PME: Thessaloniki, Greece, 2009; Volume 2, pp. 89-96.

46. Olivero, F. Conjecturing in Open-Geometric Situations in Cabri-Geometre: An Exploratory Classroom Experiment. In Proceedings of the British Society for Research into Learning Mathematics, Lancaster, UK, 5 June 1999; Bills, L., Ed.; St Martin's University College: Lancaster, UK, 1999; Volume 19, pp. 61-66.

47. Baccaglini-Frank, A.; Mariotti, M.A. Generating conjectures in dynamic geometry: The maintaining dragging model. Int. J. Comput. Math. Learn. 2010, 15, 225-253. [CrossRef]

48. Mariotti, M.A. Images and concepts in geometrical reasoning. In Exploiting Mental Imagery with Computers in Mathematics Education; Sutherland, R., Mason, J., Eds.; Springer: Berlin/Heidelberg, Germany, 1995; Volume 128, pp. 97-116.

49. Buckley, J.; Seery, N.; Canty, D. Investigating the use of spatial reasoning strategies in geometric problem solving. Int. J. Technol. Des. Educ. 2019, 29, 341-362. [CrossRef]

50. Leung, A.; Or, C.M. From construction to proof: Explanations in dynamic geometry environment. In Proceedings of the 31st Conference of the International Group for the Psychology of Mathematics Education, Seoul, Korea, 8-13 July 2007; Woo, J.H., Lew, H.C., Park, K.S., Seo, D.Y., Eds.; PME: Seoul, Korea, 2007; Volume 3, pp. 177-184. 